R. J. Cohen and W. T. Sullivan, III, eds.

\title{
Why Astronomy Needs Low-Pressure Sodium Lighting
}

\author{
Christian B. Luginbuhl \\ United States Naval Observatory, Flagstaff Station, PO Box 1149, \\ Flagstaff AZ 86002 USA
}

\begin{abstract}
The damage to the dark-sky resource upon which groundbased astronomical observatories depend is substantial and increasing, even at what are considered premier dark-sky sites. Due to its nearly monochromatic output at the $\mathrm{Na} I$ resonance doublet near $589 \mathrm{~nm}$, extensive use of low-pressure sodium (LPS) lighting in areas near astronomical observatories offers the potential preservation and even restoration of dark skies at other wavelengths, as well as minimal impact on the lighting needs of surrounding communities. Flagstaff, Arizona, with extensive use of LPS for general area lighting, has shown that this goal can be achieved.
\end{abstract}

\section{Introduction}

In the last 50 years, the worldwide astronomical community has focussed its efforts on the establishment of observatory facilities located at carefully evaluated sites of the highest astronomical quality. These efforts have been driven by two factors - the value of good site conditions to the quality of the data obtained, and increasing light pollution at existing observatory sites.

But today we find nearly all of these premier astronomical sites are either suffering degradation in their dark-sky conditions, or are threatened with such degradation. Despite their remote locations, the areas near the sites attract settlement because of favourable weather and climate conditions. Ironically, the very qualities that make them astronomically valuable lead to above average population and lighting increases. Further, there is a general trend of increased per capita use of outdoor lighting despite energy costs and concerns about global warming.

Arizona, site of Kitt Peak, Mount Hopkins, Mount Graham, and other quality astronomical sites, is experiencing a sustained population growth in excess of three percent per year, two-thirds arising from migration; this should be compared to the average U.S. growth of less than $1 \%$ per year for the past twenty years. The Big Island of Hawai'i, home to Mauna Kea, is experiencing growth in excess of two percent per year. The Kailua-Kona coast of the Big Island, where generally clear conditions allow lighting to have the greatest effect on the observatories, is the area where most of this growth is concentrated, and thus the effective growth rate is even higher than the average for the whole island. 


\section{Lighting Codes}

The first line of defence for preserving dark sky over these observatories has already been as fully effected as it can be - namely, the observatories have been located where population densities are low and where high-altitude atmospheric aerosols that back-scatter artificial light are minimal. One of the principal criteria in choosing observatory sites has been the darkness of the sky. Though other conditions affecting the quality of the site such as cloud cover and airflow can not be affected by civil regulation, the sky will not remain dark at these sites without proactive involvement of the astronomical community in lighting use policies of nearby communities.

To minimize brightening of the night sky over observatory sites, civil regulations restricting wasteful and unnecessarily polluting lighting practices must be pursued. Outdoor lighting codes, pioneered by Arizona communities such as Tucson and Flagstaff but in place now in many localities in the U.S., have been actively pursued in many locations. Their effectiveness at reducing the rate of increase of sky brightness is likely to be substantial, although uncertain.

Limited data indicate that the actual effectiveness of lighting codes in limiting sky brightness increases is uncertain. One reason is that there are problems in enforcing lighting codes, and the degree of compliance has not been determined. Pilachowski et al. (1989) have presented B and V measures of zenith sky brightness over Kitt Peak for 1987, finding an increase over "natural dark" of only 0.07 and $0.08 \mathrm{mag} / \operatorname{arcsec}^{2}$ at $\mathrm{V}$ and $\mathrm{B}$ respectively (Table 1). By comparing these measures to Garstang's "standard model" prediction (Garstang 1989) of $0.11(\mathrm{~V})$ and $0.07(\mathrm{~B}) \mathrm{mag} / \mathrm{arcsec}^{2}$, they offer evidence that the Tucson and Pima County outdoor lighting codes are having a measureable effect. But measures at nearby Mount Hopkins during the 1996 solar minimum apparently show the opposite, with measured values two to three times higher than Garstang's prediction.

Table 1. Predicted and Observed B and V Zenith Sky Brightness Increases (relative to a natural sky) at Selected U.S. Observatory Sites

\begin{tabular}{lccccc} 
& & \multicolumn{2}{c}{$\Delta \mathrm{V}$} & \multicolumn{2}{c}{$\Delta \mathrm{B}$} \\
\hline Site & Established & $\mathrm{Pre}^{a}$ & $\mathrm{Obs}^{b}$ & Pre $^{a}$ & $\mathrm{Obs}^{b}$ \\
\hline Kitt Peak & 1958 & 0.13 & 0.07 & 0.09 & 0.08 \\
Mt Hopkins / MMT & 1968 & 0.21 & 0.49 & 0.18 & 0.63 \\
Mt Graham & 1995 & 0.06 & & 0.05 & \\
Lowell / Anderson Mesa & 1963 & 0.27 & & 0.28 & \\
Naval Obs. Flagstaff & 1954 & 0.39 & 0.45 & 0.42 & 0.13 \\
McDonald & 1969 & 0.01 & & 0.01 & \\
Mauna Kea & 1970 & 0.03 & & 0.03 &
\end{tabular}

\footnotetext{
${ }^{a}$ Predicted values for 1995 from Garstang (1989), except for 1987 for Kitt Peak

${ }^{b}$ Measured values for Kitt Peak from 1987 (Pilachowski et al. 1989); Mt Hopkins for 1996

(Caldwell et al. 1999); and Naval Obs., Flagstaff, for 1996 (by the author)
} 
The Tucson and Pima County lighting code focuses attention on the fraction of uplight permitted from lighting fixtures, requiring that most lighting fixtures above a certain lumen output project no light above the horizontal plane. There is no overall limitation on the amount of lighting permitted.

Recent efforts, pioneered in Flagstaff and Coconino County, Arizona in 1989 updates to their lighting codes, and now on the verge of adoption in Tucson, include in addition overall lighting caps expressed in lumens per acre or hectare. Again, the lumens per acre caps must be having an effect from simple comparison of uncapped vs. capped lighting practices, but the overall effects have not been measured (but see Luginbuhl 2001).

Though these efforts can decrease uplight per capita, sometimes by large factors, the inexorable increase in population near observatory sites means that the sky will continue to brighten even in areas with up-to-date and effectively implemented lighting codes. Though with slow replacement of old non-conforming lighting systems there would seem some hope to actually decrease sky brightness, the limited effect of any replacement is swamped by population growth and increased amounts of lighting per capita. Near premier astronomical facilities, a further approach offers an avenue to substantially decrease the impact of lighting at most wavelengths - namely, the specification of low-pressure sodium lighting.

\section{Low-Pressure Sodium Lighting}

Low-pressure sodium (LPS) lighting produces light by passing an electrical arc through sodium vapour at low pressure, typically $5 \times 10^{-3}$ torr. The preponderance of LPS lamp emission is at the resonance doublet of $\mathrm{Na}$ I at $589.0 \mathrm{~nm}$ and $589.6 \mathrm{~nm}$, making LPS lamps nearly monochromatic.

\subsection{Astronomical Value}

Extensive use of LPS lighting in areas near astronomical facilities offers the prospect of concentrating the sky-brightening impacts to the sodium $\mathrm{D}$ lines, decreasing the brightening at other wavelengths. The upper two panels of Figure 1 show the emission spectra of the two lamp types most commonly used for outdoor lighting, high-pressure sodium (HPS) and metal halide (MH). Both HPS and $\mathrm{MH}$ exhibit complex spectra with continuum and emission line components spread across the entire visible spectrum.

In the broad- and intermediate-band photometric systems indicated in the lower panels of Figure 1, HPS and MH contaminate all passbands, whereas LPS lamps can be seen to contaminate none (Strömgren), one (SDSS) or two (Johnson-Cousins) of the passbands. The implications for observations in these systems is obvious. Recent measures of sky brightness at the Flagstaff Station (see Table 1) show that while the $\mathrm{V}$ sky brightness increase is approximately as predicted by Garstang (1989) based on population and non-LPS lighting, in B it is less than one-third the predicted value, verifying the practical effectiveness of the Flagstaff LPS requirements.

For spectroscopic observation, the implications are more severe, since the contaminating spectrum of HPS and especially MH are not only broad-spectrum but also extremely complex functions of wavelength. Accurate flux calibration 


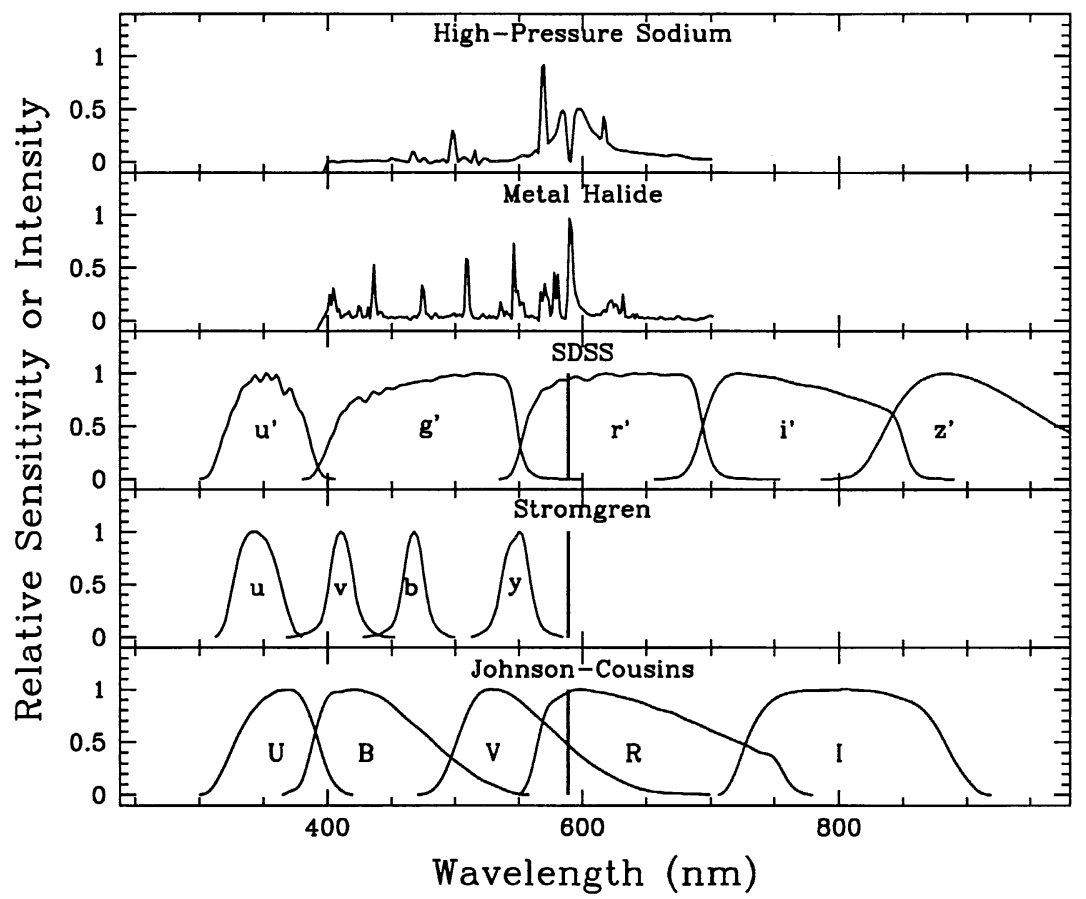

Figure 1. Relation of high-pressure sodium, metal halide and lowpressure sodium emission (vertical bar at $589 \mathrm{~nm}$ in the lower three panels) to three photometric filter systems. Only a portion of the emission spectrum of HPS and MH lamps is shown.

against such complex backgrounds is difficult, and slight but common errors will lead to spurious spectral features.

\subsection{Issues in LPS Use}

The astronomical community has much to gain from increased use of LPS lighting. However, LPS suffers a poor image, particularly within the American lighting industry. This lack of support stems principally from the poor colour rendition and the impression among some that LPS light provides for inferior visibility compared to broad-spectrum sources. There has also been considerable misinformation promulgated about LPS lighting, and the damage done to its image has been substantial. As for many other aspects of outdoor lighting and issues of visibility, safety and utility, more research is needed in some aspects of LPS lighting, such as lamp lifetimes, optimal operating conditions, and system operating costs. But the advantages of LPS are sufficiently well documented to justify its more widespread use in both astronomical and non-astronomical communities. Two of the issues concerning LPS are briefly discussed below. 
Colour Perception. Since LPS is a nearly monochromatic source, the eye cannot discriminate colours under pure LPS lighting. But colour perception is not necessary in many outdoor situations, or may be provided by other types of lighting. Many lighting codes distinguish so-called "general illumination" or "Class 2" applications where colour perception is not required. The principal Class 2 examples are roadway and parking lot lighting, and the proportion of outdoor lighting devoted to such uses is large. In Flagstaff, where LPS is strongly encouraged for Class 2 lighting, a sample of five recent development projects shows an average of $82 \%$ of outdoor lighting as LPS.

In many cases small amounts of broad-spectrum light will be available either intentionally or accidentally, such as from automobile headlights on roadways. Boynton and Purl (1989) have demonstrated that the addition of as little as $10 \%$ white light to otherwise pure LPS illumination can render essentially normal colour perception.

Efficiency and Energy Use. Since the wavelength of the principal LPS emission $(589 \mathrm{~nm})$ is so near the eye's peak photopic sensitivity (at $555 \mathrm{~nm})$, LPS has a great natural efficiency advantage. The advantage is further extended by the maintenance of this output during the operating life of the lamps. Whereas MH and HPS lamps dim considerably during use (to about $64 \%$ and $73 \%$ of initial outputs, respectively), LPS loses little or none of its initial output during its operating life. Some of this advantage is lost in practice, through slightly higher ballast losses and slightly lower efficiencies in some applications, but in most roadway and large area illumination the LPS efficiency advantage remains. Table 2 shows maintained effective lumens per watt for MH, HPS and LPS systems optimized for roadway and parking lot illumination. These figures were calculated by the author using software provided by lighting manufacturers, and use actual luminaires and other factors as summarized in the caption. For the narrowest roadway considered, LPS is $7 \%$ more efficient than HPS, and $61 \%$ more efficient than MH. The figures for parking lots are actually upper limits, assuming no edge losses, but here the efficiency of LPS towers over HPS and MH systems by $48 \%$ and $119 \%$ respectively.

In recent years the lighting profession has become aware of the scotopic or "dark-adapted" response of the eye. At low light levels the eye's peak sensitivity shifts toward the blue, with completely scotopic response peaking at about $510 \mathrm{~nm}$. If this sensitivity is the correct one to apply to outdoor lighting conditions, then the effective output of LPS is lowered because the emission is considerably removed from the peak sensitivity. The best work currently available to address the question of which sensitivity is most appropriate under typical outdoor lighting conditions (Adrian 1997) indicates that this effect is relatively minor, but much remains to be learned concerning appropriate metrics of visibility, including peripheral vision questions. For now the indication is that LPS maintains its advantage over MH and HPS under even the lowest levels of lighting recommended by the Illuminating Engineering Society of North America. 
Table 2. Application Efficiencies ${ }^{a}$ (in effective maintained lumens per watt) for metal halide (MH), high-pressure Sodium (HPS), and lowpressure sodium (LPS) lighting systems evaluated by the author.

\begin{tabular}{|c|c|c|c|}
\hline \multirow[t]{2}{*}{ Lamp Type } & \multicolumn{3}{|c|}{ Application Efficiency $(\mathrm{lm} / \mathrm{W})$} \\
\hline & 38' Road & 102' Road & Parking \\
\hline Metal Halide & 18 & 27 & 31 \\
\hline High Pressure Sodium & 27 & 41 & 46 \\
\hline Low Pressure Sodium & 29 & 50 & 68 \\
\hline
\end{tabular}

\footnotetext{
${ }^{a}$ Figures include circuit losses, light loss factors (MH: 0.54; HPS: 0.62; LPS: 0.95), and application Coefficients of Utilization (MH\&HPS: $0.45 / 0.67 / 0.75$, LPS: $0.31 / 0.54 / 0.73$ for $38^{\prime} / 102$ ' $/ \mathrm{PL}$ applications) for comparable maintained illumination level (using 175W MH, 150W HPS and 90W LPS). Luminaires used were MH\&HPS: GE M250-A2/GE M250-A2/GE Dimension, LPS: Gardco LPSA/LPSA/LPSA.
}

\section{Conclusions}

With continued growth in areas near astronomical facilities, prospects for the long-term preservation of dark skies at these sites are dim without involvement of the astronomical community in the effort to enact and enforce strict lighting ordinances. Though lighting codes with shielding standards and lumens per hectacre limits can subtantially slow the deterioration, they are unlikely to stop it. Use of low-pressure sodium (LPS) lighting offers a realistic prospect of nearly dark skies at most wavelengths, even in proximity to substantial population centers. LPS can provide effective lighting for the lowest energy expenditure of all available lighting sources, and it should be more widely used. In Flagstaff, Arizona, lighting ordinances requiring extensive use of LPS have been in effect for over ten years. While recent measures of the sky brightness at $\mathrm{V}$ indicate brightening in accord with predictions based on population and non-LPS lighting types, the increase at $B$ is less than one third the predicted value, indicating that such ordinances can be effective.

\section{References}

Adrian, W. 1997, paper given at the International Lighting Conference, Durban, South Africa.

Boynton, R. M. and Purl, K. F. 1989, Lighting Res. Technol., 21, 23

Caldwell, N., et al. 1999, personal communication.

Garstang, R. H. 1989, Ann. Rev. Astron. Astrophys., 27, 19

Luginbuhl, C. B. 2001, these proceedings

Pilachowski, C. A., et al. 1989, PASP, 101, 707 